\title{
An approach to the research on ion and water properties in the interphase between the plasma membrane and bulk extracellular solution
}

\author{
Hiroshi Hibino ${ }^{1,2,3} \mathbb{D} \cdot$ Madoka Takai $^{4} \cdot$ Hidenori Noguchi $^{5} \cdot$ Seishiro Sawamura $^{1} \cdot$ \\ Yasufumi Takahashi $^{6,7} \cdot$ Hideki Sakai $^{8} \cdot$ Hitoshi Shiku'
}

Received: 7 September 2016/Accepted: 5 February 2017/Published online: 17 February 2017

(C) The Physiological Society of Japan and Springer Japan 2017

\begin{abstract}
In vivo, cells are immersed in an extracellular solution that contains a variety of bioactive substances including ions and water. Classical electrophysiological analyses of epithelial cells in the stomach and small intestine have revealed that within a distance of several hundred micrometers above their apical plasma membrane, lies an extracellular layer that shows ion concentration gradients undetectable in the bulk phase. This "unstirred layer", which contains stagnant solutes, may also exist
\end{abstract}

Hiroshi Hibino

hibinoh@med.niigata-u.ac.jp

1 Department of Molecular Physiology, Niigata University School of Medicine, 1-757 Asahimachi-dori, Chuo-ku, Niigata, Niigata 951-8510, Japan

2 Center for Transdisciplinary Research, Niigata University, Niigata, Niigata 950-2181, Japan

3 AMED-CREST, AMED, Niigata, Japan

4 Department of Bioengineering, School of Engineering, The University of Tokyo, Tokyo, Japan

5 International Center for Materials Nanoarchitectonics and Global Research Center for Environment and Energy Based on Nanomaterials Science, National Institute for Materials Science, Tsukuba, Ibaraki, Japan

6 Division of Electrical Engineering and Computer Science, Kanazawa University, Kakuma-machi, Kanazawa 920-1192, Japan

7 Precursory Research for Embryonic Science and Technology (PRESTO), Japan Science and Technology Agency (JST), Saitama 332-0012, Japan

8 Department of Pharmaceutical Physiology, Graduate School of Medicine and Pharmaceutical Sciences, University of Toyama, Toyama, Japan

9 Graduate School of Engineering, Tohoku University, Sendai 980-8579, Japan between the bulk extracellular solution and membranes of other cells in an organism and may show different properties. On the other hand, an earlier study using a bacterial planar membrane indicated that $\mathrm{H}^{+}$released from a transporter migrates in the horizontal direction along the membrane surface much faster than it diffuses vertically toward the extracellular space. This result implies that between the membrane surface and unstirred layer, there is a "nanointerface" that has unique ionic dynamics. Advanced technologies have revealed that the nanointerface on artificial membranes possibly harbors a highly ordered assembly of water molecules. In general, hydrogen bonds are involved in formation of the ordered water structure and can mediate rapid transfer of $\mathrm{H}^{+}$between neighboring molecules. This description may match the phenomenon on the bacterial membrane. A recent study has suggested that water molecules in the nanointerface regulate the gating of $\mathrm{K}^{+}$channels. Here, the region comprising the unstirred layer and nanointerface is defined as the interphase between the plasma membrane and bulk extracellular solution (iMES). This article briefly describes the physicochemical properties of ions and water in the iMES and their physiological significance. We also describe the methodologies that are currently used or will be applicable to the interphase research.

Keywords Unstirred layer - Nanointerface · Bulk extracellular fluid $\cdot$ Ions $\cdot$ Membrane $\cdot$ Water

\section{Introduction}

All cells in multicellular organisms are separated from the extracellular fluid by the plasma membrane, which is composed of a lipid bilayer. The membrane not only 
encloses organelles, molecules, enzymes, and genes inside the cells but also contains receptors and the ion transport apparatus including channels and transporters. To date, the majority of experimental techniques have been designed to analyze the intracellular compartment, plasma membrane, and bulk extracellular fluid. These conventional approaches have identified numerous biological events and their underlying mechanisms, greatly contributing to the development of life sciences and medicine. Nevertheless, currently available data do not sufficiently explain the machineries that yield a considerable number of vital phenomena such as stable extracellular and intracellular ionic homeostasis, effective absorption of nutrients and drugs, and proper control of ligand-receptor interactions. To resolve this problem, different methodological approaches are required. Several studies have focused on the microextracellular space above the plasma membrane. The fluid in this space is not readily mixed with bulk content and likely maintains particular physicochemical profiles of ions and water. This space, which is here referred to as the "interphase between the membrane and bulk extracellular solution (iMES)", can be subdivided into two regions as follows (Fig. 1). First, most of the iMES is occupied by an "unstirred layer", which contains stagnant solutes [1]. This layer may be comparable to the "diffusion layer" described by other scientists [2] and is unlikely to be present in the narrow intercellular space between the membranes of tightly attached or aggregated cells. Second, between the unstirred layer and membrane, in other words, in the very

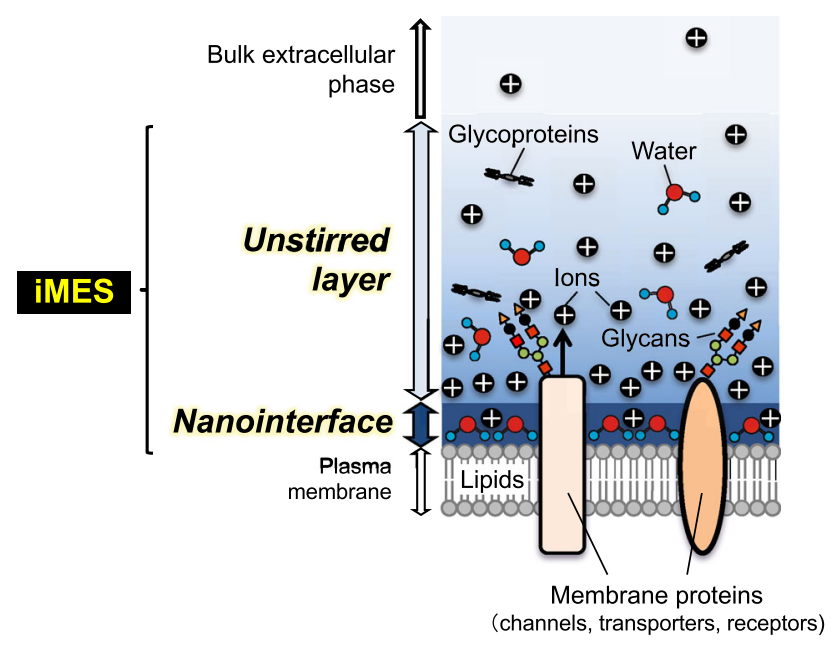

Fig. 1 Structure of the iMES. The iMES is defined as a complex of the "unstirred layer" and "nanointerface." Substances and components that constitute the layers are illustrated. They include ions, structurally unique water molecules, extracellular regions of membrane proteins and their glycans, and secretory glycoproteins. Of note, the nanointerface lies in close vicinity of the membrane surface and possibly has thickness ranging within a nanometer. For the characteristics of the unstirred water layer and nanointerface, see the main text close vicinity to the membrane surface-possibly within less than a nanometer from the outer leaflet-lies a space that is likely to be characterized by water structure and ionic dynamics different from those in the unstirred layer. Here, we call this space the "nanointerface", and it may cover the plasma membrane of all the cells. In spite of potential importance of the iMES in organisms, it has not yet been sufficiently examined by conventional methods.

The iMES does not perfectly correspond to a "biointerface" or "biological interphase" that is likely to include the cellular plasma membrane in addition to the unstirred layer and a nanointerface as documented in the literature [2]. In this review, we focus on ions and water in the iMES and briefly summarize their unique properties and possible physiological significance. Moreover, we describe several techniques and methodologies that have been applied or will be applicable to analysis of the iMES. Some of the topics were presented at the symposium "The 93rd Annual Meeting of the Physiological Society of Japan" (March 22-24, 2016).

\section{Unstirred layer}

The unstirred layer, which is mostly observed above the epithelial apical membranes exposed to an extracellular fluid in a lumen, contains not only solutes including ions, secretory proteins, and peptides, but also structural elements such as glycans and extracellular regions of ion channels, transporters, and receptors (Fig. 1) [2, 3]. This region is likely to serve as a diffusion barrier, allowing ions to display physicochemical behaviors that are undetectable in the bulk phase [4-6]. In this context, the $\mathrm{H}^{+}$ parameter in the digestion system has been characterized well. In the stomach, a layer of $100-$ to $300-\mu \mathrm{m}$ thickness above the plasma membrane of the epithelial cells has a $\mathrm{pH}$ gradient from 2 to 7 [7]. This interphase protects the epithelial surface from damage by the strong acid $(\mathrm{pH} \approx 1)$ of the bulk fluid. The small intestine has a $\sim 500-\mu \mathrm{m}$ unstirred layer, which has $\mathrm{pH}$ of 6-7 and shields the epithelial cells from enteral contents whose $\mathrm{pH}$ is approximately neutral [7-9]. The cells take up nutrition via membrane-integral transporters that carry $\mathrm{H}^{+}$and peptides together. The excess $\mathrm{H}^{+}$in the layer likely acts as a driving force for the transporters and facilitates absorption of nutrition. These $\mathrm{pH}$ properties seem to be maintained at least partially by the convoluted surface of the mucosa, the glycans attached to membrane proteins, and the mucus $[3,6]$.

It is reasonable to hypothesize that the unstirred layer may cover a variety of cells including floating immune cells and scatteredly distributed fibroblasts in an organism. In this layer, not only $\mathrm{H}^{+}$but also other ions may show 
profiles different from those in the bulk phase. This idea is partially supported by one study involving an artificially fabricated lipid-bilayer membrane [10]. In that study, two chambers, each of which contained any one or two of $\mathrm{K}^{+}$, $\mathrm{Na}^{+}, \mathrm{H}^{+}$, and $\mathrm{Ca}^{2+}$ and the corresponding ionophore(s), were separated by the membrane. The ion concentrations in one chamber were set to greatly exceed those in the other. As a consequence, ion concentration gradients were constantly detected in the space located within several hundred micrometers from the membrane surface, reminiscent of the unstirred layer. Thickness of the space depends on the magnitude of the diffusion coefficient of an ion. In vivo, individual cell types have different sets of ion transport machinery, and therefore may have an unstirred layer of variable properties and thickness. It will be worthwhile to elucidate how different profiles of the unstirred layer are established and how they influence cellular and tissue functions.

To vertically analyze concentration gradients of ions in the unstirred layer, glass microelectrodes are conventionally used whose tips are packed with ion exchangers. Each exchanger type selectively allows certain ion species to pass through. In a typical case, microelectrodes with a tip diameter of $1-2 \mu \mathrm{m}$ are manufactured and gradually brought close to the membrane surface by a manipulator $[8,10]$. A disadvantage of this classical method is difficulty with planer mapping of ionic concentrations. This problem has not yet been addressed sufficiently but may be resolved in the future by application of a recently developed scanning ion-conductance microscopy (SICM). This method, which was originally aimed at noninvasive visualizing of fine three-dimensional (3D) structure of the surface of live cells, involves two $\mathrm{Ag} / \mathrm{AgCl}$ electrodes. One is inserted into the probe of a fine glass nanopipette filled with an electrolyte, and the other is placed in the medium (Fig. 2a) $[11,12]$. A voltage is applied between the two $\mathrm{Ag} / \mathrm{AgCl}$ electrodes to monitor the ion current that represents a distance between an electrochemical probe and the sample. As the nanopipette gets closer to the surface of the cell (Fig. 2a), the access resistance increases, reducing the ion current from the electrode (Fig. 2b). The hopping probe scans the cell surface, while the reduction in the current is continuously monitored (Fig. 2a). Analysis of the positions of the tip of the probe at certain set points of the current leads to construction of a $3 \mathrm{D}$ image of the surface on a nanometer scale on the computer (Fig. 2c) [12-14]. SICM can precisely measure the distance between the tip of the electrode and the cell surface. If a double-barreled microelectrode, where each barrel has either an ion-selective electrode or SICM electrode, were prepared and equipped with the aforementioned manipulation and monitoring system, then 3D mapping of ionic distribution in the unstirred layer could be achieved at high resolution.
To visualize the ionic properties, other powerful tools must also be used: a variety of bioimaging probes including $\mathrm{Ca}^{2+}$ indicators such as fluo-4 and ratiometric fura- $2, \mathrm{pH}$ sensing 2',7'-Bis-(2-carboxyethyl)-5-(and-6-)carboxyfluorescein $(\mathrm{BCECF}), \mathrm{Na}^{+}$-sensing sodium-binding benzofuran isophthalate $(\mathrm{SBFI})$, and $\mathrm{K}^{+}$-detecting potassium-binding benzofuran isophthalate (PBFI) [15-18]. Nevertheless, a special modification of the probes will be necessary to avoid their diffusion to the bulk phase. To address this issue, a basic technology has been developed by researchers including one of the authors of the present article [19]. They conjugated rhodamine nanoparticles to lectin, which has high affinity for sialic acid residues (Fig. 3a). Because these residues are located at the distal end of glycans of the membrane proteins, the fluorescent probes remain in the unstirred layer. A recent work has shown that the lectin-conjugated probe clearly labels sialic acids of the $\mathrm{H}^{+}, \mathrm{K}^{+}$-ATPase $\beta$-subunit stably expressed together with the $\alpha$-subunit in live LLC-PK1 cells (Fig. 3b) [20]. This tool may also help to visualize the distribution of mucus that contains numerous glycans. Moreover, replacing rhodamine with other compounds may enable us to fabricate imaging probes for monitoring of different ions in the unstirred layer.

\section{Nanointerface}

In the nanointerface that lies immediately above the membrane surface, unusual structure of water molecules has been recently demonstrated by advanced technologies such as sum frequency generation (SFG) spectroscopy and atomic force microscopy (AFM). SFG is a second-order nonlinear optical process, in which two photons of frequencies $\omega_{1}$ and $\omega_{2}$ generate one photon of sum frequency $\left(\omega_{\mathrm{SFG}}=\omega_{1}+\omega_{2}\right)[21,22]$ (Fig. 4a). By means of visible light of fixed wavelength and tunable infrared light as two input light sources, the SFG signal is resonantly enhanced when the energy of the infrared beam becomes equal to that of a vibrational state of surface species. SFG spectroscopy is ineffective in a medium with inversion symmetry under the electric dipole approximation and is thus allowed only at the interface where the inversion symmetry is necessarily broken. Thus, an SFG signal can be a surface-sensitive form of vibrational spectroscopy. Theoretically, this method can detect the profile of submonolayer structures of the molecules in the interface [22]. The properties of a water molecule were examined by SFG spectroscopy in the interface between the medium and the substrate coated by a polymer brush, highly condensed polymer chains like a forest [23] (Fig. 4a). The results indicate that when 2-methacryloyloxyethyl phosphorylcholine (MPC) polymers, which mimic phosphorylcholine groups of the 
a
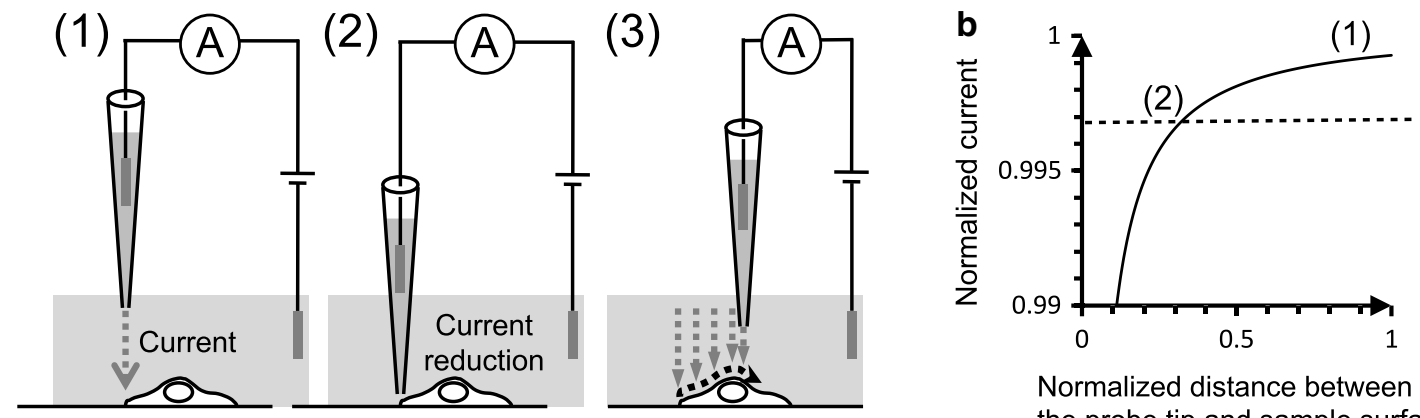

Normalized distance between the probe tip and sample surface

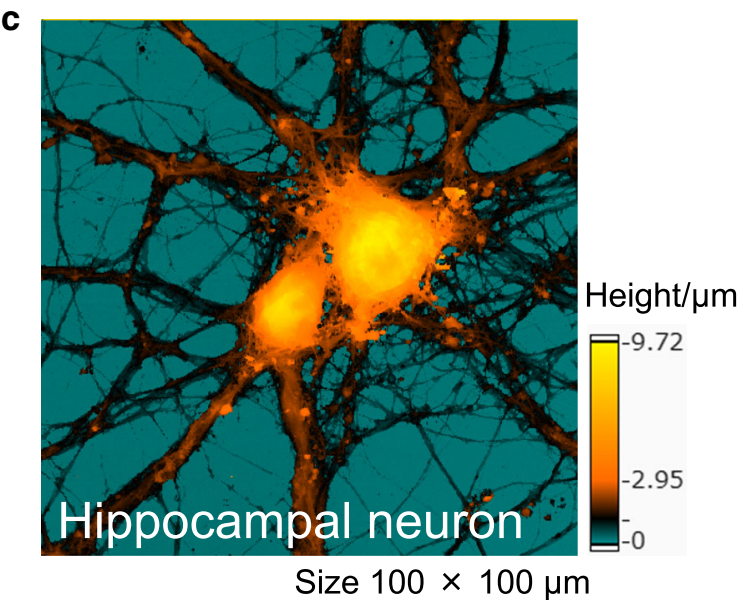

Fig. 2 Scanning ion-conductance microscopy (SICM). a, b The operating principle of SICM. A nanopipette, which is provided with a voltage, approaches the surface of the cell, as shown in panel (1) of a. In this process, the current begins to decrease (b). The nanopipette is stopped when the current reaches a certain value [panel (2) in a and point (2) in b]. This step is repeated multiple times; the cell surface is scanned with the probe to create an image on the computer. For details, see the main text. In $\mathbf{b}$, the current was normalized to the bulk

cellular plasma membrane, are used as the material, the water molecules in the interface strongly interact with the brush (Fig. 4b). Furthermore, the structure of the water molecules is likely to depend on the types and concentrations of phospholipids constituting the brush [23].

Water structure in the nanointerface is also investigated by frequency modulation-AFM (FM-AFM) that employs a sharp cantilever with a tip diameter of several nanometers as a force sensor. In this method, a piconewton force can be detected at subnanometer spatial resolution. This excellent performance has enabled visualization of a distribution of water with its one layer sensitivity at the interface as follows [24]. Fukuma et al. analyzed water properties in the interface on a dipalmitoylphosphatidylcholine (DPPC) bilayer using FM-AFM and revealed two hydration layers with marked force emergence. The precise 3D analyses of these layers then visualized their corrugations, which likely mirror the architecture of the lipid headgroups of DPPC. This patterned

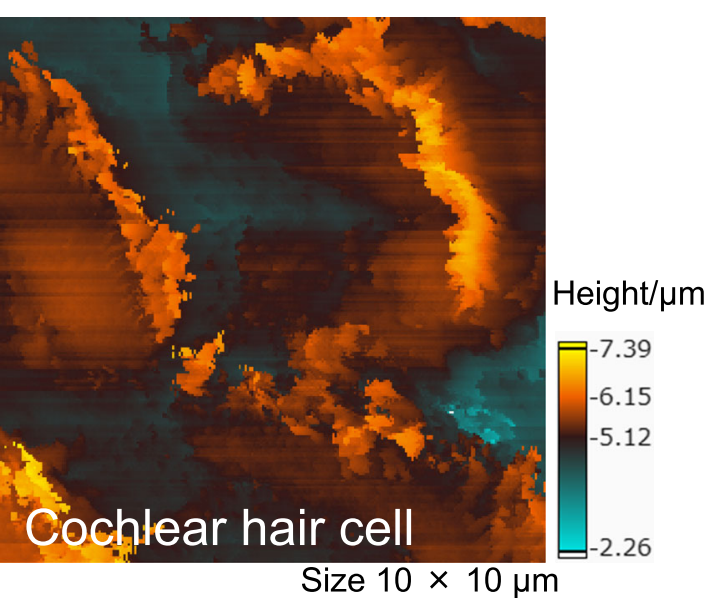

steady-state tip ion current, whereas the distance between the probe's tip and sample's surface was normalized to the inner radius of the tip. c Images obtained by SICM. Surface morphologies of hippocampal neurons of a rat and cochlear hair cells of a guinea pig were examined by SICM and displayed in left and right panels, respectively. The experiments were performed using protocols published elsewhere $[12,13,14]$

structure was stably detectable throughout the membrane. The authors suggest that the hydration layers can serve as an energy barrier for the ions and proteins approaching the membrane as well as a component controlling their physicochemical dynamics in the interface [24].

Identification of the strong interaction of water molecules with the MPC polymer brush by SFG spectroscopy and detection of the particular hydration layers on a DPPC membrane by FM-AFM imply existence of a highly ordered water structure in the nanointerface (see Fig. 4a). This profile may be involved in interfacial ion dynamics, which is clearly different from the unstirred-layer's physicochemical properties governed primarily by a diffusion process. Heberle et al. extracted a planar membrane expressing a $\mathrm{H}^{+}$secreting transporter, bacteriorhodopsin, from Halobacterium salinarum and examined the behavior of the ion released from the transporter. In this assay, Cys36, a residue localized in the transporter's intracellular loop in close 

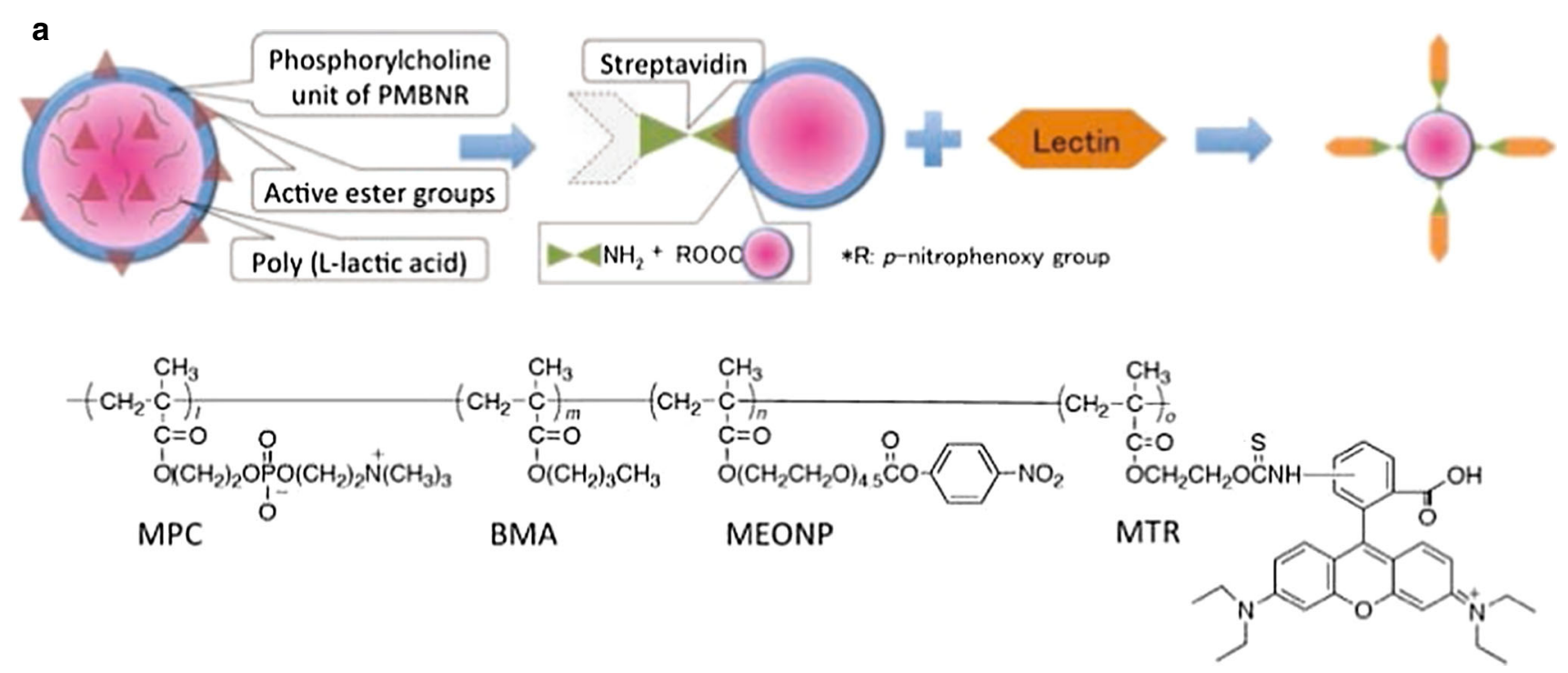

b

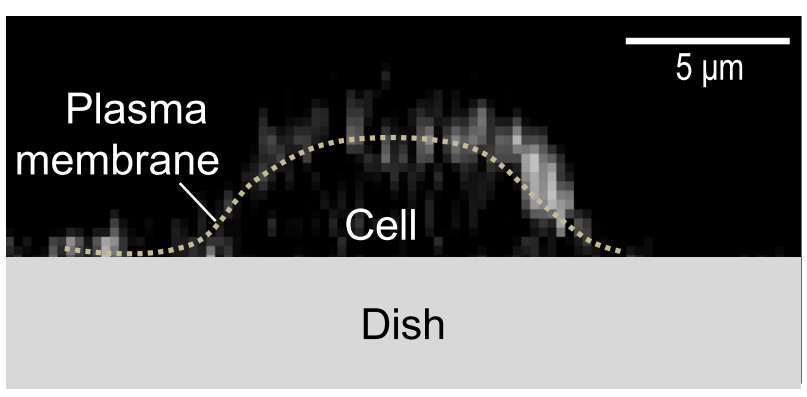

Imaging probe

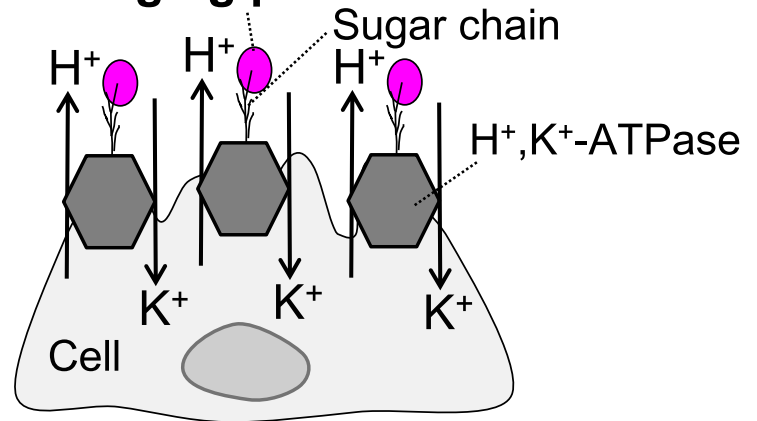

Fig. 3 A bioimaging probe targeting the interphase. a The design of fluorescent probes that specifically detect a sialic acid residue. The manufacturing process is schematically described in the upper panel. Nanoparticles were composed of 2-methacryloyloxyethyl phosphorylcholine (MPC)-based polymers namely poly(MPC)-co-n-butyl methacrylate (BMA)-co-o-nitrophenyloxycarbonyl polyethylene glycol methacrylate (MEONP)-co-p-methacryloxyethyl thiocarbonyl rhodamine B (MTR). This PMBNR probe is biocompatible because of the coating with MPC polymers. Reproduced from [19]. Reprinted

vicinity of the outer leaflet of the membrane, was bound to an $\mathrm{H}^{+}$-sensitive probe, fluorescein, whereas the extracellular solution exposed to the membrane also contained the other probe, pyramine. $\mathrm{H}^{+}$released in response to illumination migrates to the bulk extracellular compartment as well as along the outer leaflet and then inner leaflet of the bacterial planar membrane. Monitoring of the change of light intensity of the two different probes revealed that the released $\mathrm{H}^{+}$ diffused along the membrane surface much faster than toward the bulk phase [25]. Similar rapid $\mathrm{H}^{+}$movement is also observed in ice, which consists of water molecules assembled with highly ordered hydrogen bond (H-bond) networks. In this solid, $\mathrm{H}^{+}$can be displaced between neighboring water molecules within the H-bond networks and therefore move much faster than in the liquid [26]. This with permission from Cho et al. [19]. Copyright 2014 American Chemical Society. b Visualization of sugar chains in the interphase. LLC-PK1 cells coexpressing $\alpha$ and $\beta$ subunits of $\mathrm{H}^{+}, \mathrm{K}^{+}$-ATPases were treated with the fluorescent probes described in $\mathbf{a}$. Note that the $\beta$ subunit is glycosylated and contains sialic acid groups. Left panel shows a representative example of a vertical scan image of the cell. Right panel is a schematic illustration demonstrating the binding of the probes to the sugar chains of the $\beta$ subunit

"proton hopping" mechanism may account for the high speed of the lateral diffusion of $\mathrm{H}^{+}$on the bacterial planar membrane because its nanointerface is expected to harbor highly ordered water molecules, as in the aforementioned data from SFG spectroscopy and FM-AFM [27, 28]. Further studies will be needed to validate this hypothesis.

Functional importance of water molecules in the nanointerface has been ascribed to the gating of $\mathrm{K}^{+}$channels, as reported by Syeda et al. [29]. In that study, the pore structure without the voltage sensor was reconstituted in lipid bilayers, and the channel activity was measured at different compositions of lipids. When phosphoglycerides located on the extracellular leaflet contained glycerol or inositol, the channel conductance was reduced and the opening time was prolonged. The mechanisms underlying 

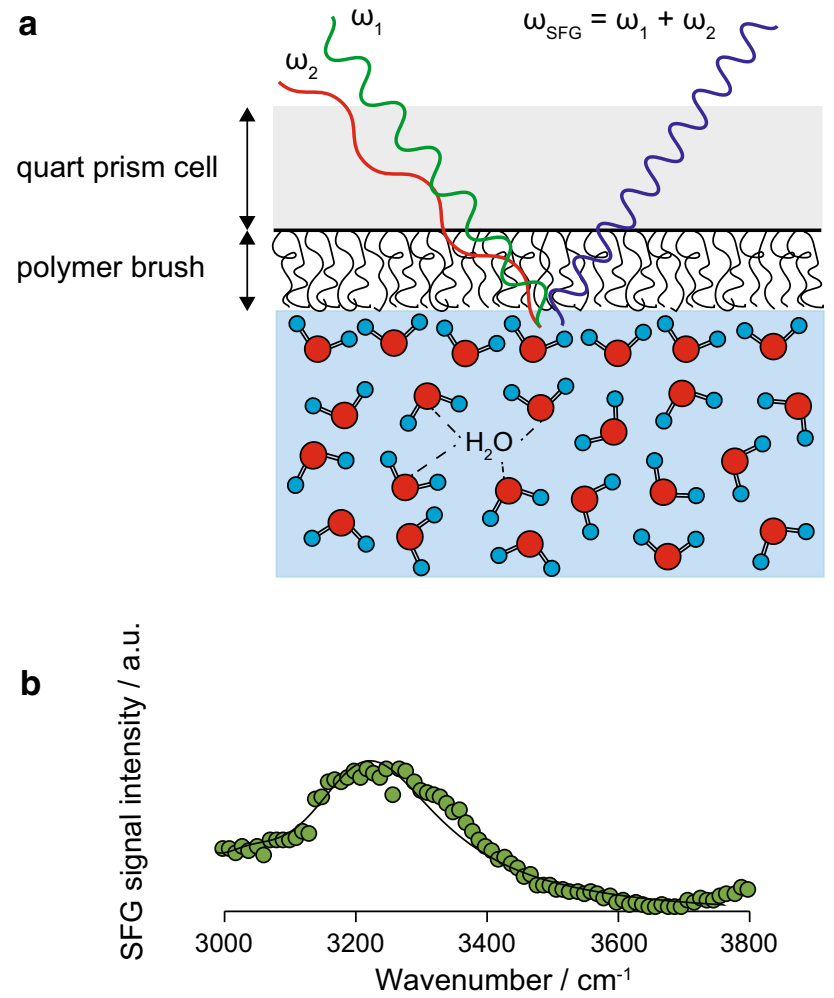

Fig. 4 Sum frequency generation (SFG) spectroscopy. a SFG spectrometry. In the illustrated experiment, water-immersed polymer brush is examined, a dense collection of polymer chains on the surface of a quart prism cell. When two photons of frequencies $\left(\omega_{1}\right.$ and $\omega_{2}$ ) were simultaneously provided for the sample, the SGF signal $\left(\omega_{\mathrm{SFG}}=\omega_{1}+\omega_{2}\right)$ can emerge from water molecules whose inversion symmetry is broken. It seems probable that the nanointerface contains a number of these molecules. Note that the signal would not be generated from the water molecules that show random orientations. b SFG spectra of the $\mathrm{OH}$ stretching region of the MPC polymer brush using isotopic dilution water $\left(\mathrm{HOD}, \mathrm{H}_{2} \mathrm{O} / \mathrm{D}_{2} \mathrm{O}=1 / 4\right)$ [23]

these observations were proposed as follows; the presence of glycerol or inositol caused depletion of water from the exit of the ion conductance path in the channel, and this phenomenon prevented $\mathrm{K}^{+}$from exiting the filter gate from rehydration. In support of this notion, similar effects on channel activity were observed when small amounts of ethylene glycol or mannitol, which are cryoprotectants that modulate water structure by interacting with hydrogen bonds, are added to the bath solution. In any case, modulation of the channel is likely to be related to the neighboring extracellular water molecules present in the nanointerface.

\section{Perspectives}

To further study the iMES, bioanalytical technologies must be developed more actively. For example, the unstirred layer should also contain solutes such as hormones and peptides as mentioned above. For monitoring of the dynamics of these bioactive substances, electrochemical approaches may be applicable. For instance, microelectrodes composed of carbon or conductive diamond can measure concentrations of monoamines [30, 31]. Modification of the surface of the materials with particular compounds may allow the electrodes to detect specific substances in the unstirred layer. An important issue in the development of bioimaging and electrochemical probes will be to avoid their interference with the conditions and milieus of the interphase as much as possible. To this end, coating of the probe's surface with biocompatible phospholipid polymers would reduce nonspecific absorption of proteins and other substances. This modification was used for the aforementioned fluorescent nanoparticles targeting sialic acid residues, thus resulting in improved sensitivity and specificity [19].

It is also necessary to clarify how the detected physicochemical phenomena in the unstirred layer and nanointerface are regulated by membrane proteins and how they affect cellular and tissue functions. In situ and in vivo assays should be designed to directly examine the relation between events in the interphase and tissue or organ activities. Experimental and theoretical approaches using phospholipid polymer brush membranes and computational models are also needed. In particular, several simulations of macroscopic ionic dynamics based on ion channels and transporters have been recently described [32, 33]; these could be combined with calculations of microscopic ion dynamics in the iMES. Consolidation of all the data will unveil the physiological and pathological significance of the interphase. These endeavors may establish a new interdisciplinary field of research in life sciences.

Acknowledgements We would like to thank Editage (http://www. editage.jp) for English language editing. This work is partially supported by AMED-CREST, AMED (to HH).

\section{Compliance with ethical standards}

Conflict of interest None of the authors has any conflict of interest to report.

Ethical approval This article does not contain any studies with human participants or animals performed by any of the authors.

\section{References}

1. Barry PH, Diamond JM (1984) Effects of unstirred layers on membrane phenomena. Physiol Rev 64:763-872

2. van Leeuwen HP, Köster W (2004) Physicochemical kinetics and transport at biointerfaces, vol 9. Wiley, Chichester

3. Moran AP, Gupta A, Joshi L (2011) Sweet-talk: role of host glycosylation in bacterial pathogenesis of the gastrointestinal tract. Gut 60:1412-1425 
4. Lauger P (1976) Diffusion-limited ion flow through pores. Biochim Biophys Acta 455:493-509

5. Finkelstein A (1987) Water movement through lipid bilayers, pores, and plasma membranes. Wiley, New York

6. Levy MN, Koeppen BM, Stanton BA (2005) Principles of physiology, 4th edn. Mosby, Philadelphia

7. Yandrapu H, Sarosiek J (2015) Protective Factors of the Gastric and Duodenal Mucosa: an Overview. Curr Gastroenterol Rep $17: 24$

8. Shiau YF, Fernandez P, Jackson MJ, McMonagle S (1985) Mechanisms maintaining a low-pH microclimate in the intestine. Am J Physiol 248:G608-G617

9. Moran MM, McAlexander MA, Biro T, Szallasi A (2011) Transient receptor potential channels as therapeutic targets. Nat Rev Drug Discov 10:601-620

10. Pohl P, Saparov SM, Antonenko YN (1998) The size of the unstirred layer as a function of the solute diffusion coefficient. Biophys J 75:1403-1409

11. Hansma PK, Drake B, Marti O, Gould SA, Prater CB (1989) The scanning ion-conductance microscope. Science 243:641-643

12. Takahashi Y, Shevchuk AI, Novak P, Murakami Y, Shiku H, Korchev YE, Matsue T (2010) Simultaneous noncontact topography and electrochemical imaging by SECM/SICM featuring ion current feedback regulation. J Am Chem Soc 132:10118-10126

13. Takahashi Y, Shevchuk AI, Novak P, Zhang Y, Ebejer N, Macpherson JV, Unwin PR, Pollard AJ, Roy D, Clifford CA, Shiku H, Matsue T, Klenerman D, Korchev YE (2011) Multifunctional nanoprobes for nanoscale chemical imaging and localized chemical delivery at surfaces and interfaces. Angew Chem 50:9638-9642

14. Takahashi Y, Shevchuk AI, Novak P, Babakinejad B, Macpherson J, Unwin PR, Shiku H, Gorelik J, Klenerman D, Korchev YE, Matsue T (2012) Topographical and electrochemical nanoscale imaging of living cells using voltage-switching mode scanning electrochemical microscopy. Proc Natl Acad Sci 109:11540-11545

15. Terai T, Nagano T (2013) Small-molecule fluorophores and fluorescent probes for bioimaging. Eur J Physiol 465:347-359

16. Arosio D, Ratto GM (2014) Twenty years of fluorescence imaging of intracellular chloride. Front Cell Neurosci 8:258

17. Han J, Burgess K (2010) Fluorescent indicators for intracellular pH. Chem Rev 110:2709-2728

18. Meuwis K, Boens N, Gallay J, Vincent M (1998) Photophysics of Mag-fura-2: a fluorescent indicator for intracellular $\mathrm{Mg}^{2+}$. Chem Phys Lett 287:412-420

19. Cho J, Kushiro K, Teramura Y, Takai M (2014) Lectin-tagged fluorescent polymeric nanoparticles for targeting of sialic acid on living cells. Biomacromolecules 15:2012-2018

20. Fujii T, Watanabe M, Shimizu T, Takeshima H, Kushiro K, Takai M, Sakai H (2016) Positive regulation of the enzymatic activity of gastric $\mathrm{H}^{+}, \mathrm{K}^{+}$-ATPase by sialylation of its beta-subunit. Biochim Biophys Acta 1858:1228-1235

21. Du Q, Superfine R, Freysz E, Shen YR (1993) Vibrational spectroscopy of water at the vapor/water interface. Phys Rev Lett 70:2313-2316

22. Shen YR, Ostroverkhov V (2006) Sum-frequency vibrational spectroscopy on water interfaces: polar orientation of water molecules at interfaces. Chem Rev 106:1140-1154

23. Nagasawa D, Azuma T, Noguchi H, Uosaki K, Takai M (2015) Role of interfacial water in protein adsorption onto polymer brushes as studied by SFG spectroscopy and QCM. J Phys Chem C 119:17193-17201

24. Fukuma T (2010) Water distribution at solid/liquid interfaces visualized by frequency modulation atomic force microscopy. Sci Technol Adv Mater 11:033003

25. Heberle J, Riesle J, Thiedemann G, Oesterhelt D, Dencher NA (1994) Proton migration along the membrane surface and retarded surface to bulk transfer. Nature 370:379-382

26. Uritski A, Presiado I, Huppert D (2008) Indication of a very large proton diffusion in ice $\mathrm{I}_{\mathrm{h}}$. J Phys Chem C 112:11991-12002

27. Springer A, Hagen V, Cherepanov DA, Antonenko YN, Pohl P (2011) Protons migrate along interfacial water without significant contributions from jumps between ionizable groups on the membrane surface. Proc Natl Acad Sci U S A 108:14461-14466

28. Zhang C, Knyazev DG, Vereshaga YA, Ippoliti E, Nguyen TH, Carloni P, Pohl P (2012) Water at hydrophobic interfaces delays proton surface-to-bulk transfer and provides a pathway for lateral proton diffusion. Proc Natl Acad Sci USA 109:9744-9749

29. Syeda R, Santos JS, Montal M (2014) Lipid bilayer modules as determinants of $\mathrm{K}^{+}$channel gating. J Biol Chem 289:4233-4243

30. Suzuki A, Ivandini TA, Yoshimi K, Fujishima A, Oyama G, Nakazato T, Hattori N, Kitazawa S, Einaga Y (2007) Fabrication, characterization, and application of boron-doped diamond microelectrodes for in vivo dopamine detection. Anal Chem 79:8608-8615

31. Clark JJ, Sandberg SG, Wanat MJ, Gan JO, Horne EA, Hart AS, Akers CA, Parker JG, Willuhn I, Martinez V, Evans SB, Stella N, Phillips PE (2010) Chronic microsensors for longitudinal, subsecond dopamine detection in behaving animals. Nat Methods 7:126-129

32. Nin F, Hibino H, Murakami S, Suzuki T, Hisa Y, Kurachi Y (2012) Computational model of a circulation current that controls electrochemical properties in the mammalian cochlea. Proc Natl Acad Sci 109:9191-9196

33. Murakami S, Kurachi Y (2016) Mechanisms of astrocytic $\mathrm{K}^{+}$ clearance and swelling under high extracellular $\mathrm{K}^{+}$concentrations. J Physiol Sci 66:127-142 